\title{
COMPOSITIONS OF PSEUDO ALMOST AUTOMORPHIC FUNCTIONS VIA MEASURE THEORY AND APPLICATIONS
}

\author{
ZHENBIN FAN, QIXIANG DONG AND GANG LI \\ Communicated by Da Xu
}

\begin{abstract}
In this paper, we establish some composition theorems of $\mu$-pseudo almost automorphic functions via measure theory, then derive sufficient conditions for the existence and uniqueness of pseudo almost automorphic mild solutions to fractional differential equations with Caputo derivatives.
\end{abstract}

1. Introduction. In 1962, Bochner [4] introduced the concept of almost automorphic functions as a natural generalization of the classical concept of almost periodic functions. Subsequently, new concepts such as pseudo almost automorphic functions, asymptotically almost automorphic functions, Stepanov-like almost automorphic functions and Stepanov-like pseudo almost automorphic functions were developed by Liang and Xiao, N'Guérékata and Pankov, Diagana, et al., respectively. Many important and interesting properties of these functions, composition theorems and applications to differential and integral equations are established. We refer the reader to the monographs $[\mathbf{1 0}, \mathbf{2 1}, \mathbf{2 2}]$, papers $[\mathbf{1}, \mathbf{2}, \mathbf{3}],[\mathbf{5}]-[\mathbf{9}],[\mathbf{1 1}]-[\mathbf{2 0}],[\mathbf{2 6}]-[\mathbf{2 8}]$ and the references therein.

Recently, Blot, Cieutat and Ezzinbi introduced another new notion of $\mu$-pseudo almost automorphic functions, which generalizes that of weighted pseudo almost automorphic functions, by applying the measure theory in [2], and they established some basic properties of these functions and composition theorems. Moreover, Chang, Zhang and

2010 AMS Mathematics subject classification. Primary 43A60, 45N05, 47D60.

Keywords and phrases. Pseudo almost automorphic, composition, mild solution.

This work was supported by the NSF of China (grant Nos. 11571300, 11271316), the Qing Lan Project of Jiangsu Province of China and High-Level Personnel Support Program of Yangzhou University. 2016.

Received by the editors on January 20, 2015, and in revised form on February 26, 
N'Guérékata developed this method and discussed the composition theorems of $\mu$-pseudo almost automorphic functions and their applications to abstract equations in $[\mathbf{5}, \mathbf{6}]$.

Motivated by the above research, the main purpose of this paper is to again study compositions of $\mu$-pseudo almost automorphic functions under conditions which are weaker than the Lipschitz condition. Moreover, under these conditions, we investigate the existence and uniqueness of $\mu$-pseudo almost automorphic mild solutions to the abstract differential equations in a Banach space $X$ of the form

$$
D_{t}^{\alpha} x(t)=A x(t)+J_{t}^{1-\alpha} f(t, x(t)), \quad t \in \mathbb{R},
$$

where $0<\alpha<1, A: D(A) \subseteq X \rightarrow X$ generates a resolvent $S_{\alpha}(t), D_{t}^{\alpha}$ is the Caputo fractional derivative and

$$
J_{t}^{1-\alpha} h(t)=\int_{0}^{t}(t-s)^{-\alpha} h(s) / \Gamma(1-\alpha) \mathrm{d} s .
$$

Furthermore, we note that almost periodic solutions, almost automorphic and compact almost automorphic solutions to the above equation have been derived in recent papers $[\mathbf{1}, \mathbf{8}, \mathbf{9}, \mathbf{2 0}]$ by Agarwal, et al., Cuevas, et al., Cuevas and Lizama and Lizama and Poblete, respectively. Thus, our main results in this paper generalize and extend many classical results on almost automorphic functions, pseudo almost automorphic and weighted pseudo almost automorphic functions.

The paper is organized as follows. In Section 2, we give some basic definitions and results for pseudo almost automorphic functions and resolvent operators. In Section 3, we establish compositions of $\mu$-pseudo almost automorphic functions under conditions which are weaker than the Lipschitz condition. In Section 4, we derive sufficient conditions to guarantee the existence and uniqueness of $\mu$-pseudo almost automorphic mild solutions to equation (1.1).

2. Preliminaries. Throughout this paper, $p \in[1, \infty)$, and we denote by $\mathbb{N}, \mathbb{Z}$ and $\mathbb{R}$ the set of positive integers, the set of integers and the set of real numbers, respectively. Let $(X,\|\cdot\|)$ and $\left(Y,\|\cdot\|_{Y}\right)$ be two Banach spaces. Let $B C(\mathbb{R}, X)$ (respectively, $B C(\mathbb{R} \times Y, X)$ ) denote the space of bounded continuous functions with supremum norm $\|\cdot\|_{\infty}$ (respectively, the space of jointly bounded continuous functions). Moreover, we denote by $\mathscr{L}(X)$ the space of bounded linear operators 
from $X$ to $X$ endowed with the operator topology, $\mathfrak{B}$ the Lebesgue $\sigma$-field of $\mathbb{R}$ and $\mathfrak{M}$ the set of all positive measures $\mu$ on $\mathfrak{B}$ satisfying $\mu(\mathbb{R})=+\infty$ and $\mu([a, b])<+\infty$, for all $a, b \in \mathbb{R}$ with $a<b$.

Now, let us recall the following definitions and basic results about almost automorphic functions.

\section{Definition 2.1.}

(i) A continuous function $f: \mathbb{R} \rightarrow X$ is said to be almost automorphic if, for every sequence of real numbers $\left\{s_{n}^{\prime}\right\}_{n=1}^{\infty}$, we can extract a subsequence $\left\{s_{n}\right\}_{n=1}^{\infty}$ such that $g(t)=\lim _{n \rightarrow \infty} f\left(t+s_{n}\right)$ is well defined in $t \in \mathbb{R}$, and $\lim _{n \rightarrow \infty} g\left(t-s_{n}\right)=f(t)$ for each $t \in \mathbb{R}$. Denote by $A A(X)$ the set of all such functions.

(ii) A continuous function $f: \mathbb{R} \times Y \rightarrow X$ is said to be almost automorphic if $f(t, u)$ is almost automorphic in $t \in \mathbb{R}$ uniformly for all $u \in K$, where $K$ is any bounded subset of $Y$. Denote by $A A(\mathbb{R} \times Y, X)$ the set of all such functions.

Definition 2.2. A continuous function $f: \mathbb{R} \rightarrow X(\mathbb{R} \times Y \rightarrow X)$ is said to be pseudo almost automorphic if it can be decomposed as $f=g+\varphi$ where $g \in A A(X)(A A(\mathbb{R} \times Y, X))$ and $\varphi \in A A_{0}(X)\left(A A_{0}(\mathbb{R} \times Y, X)\right)$. Denote by $P A A(X)(P A A(\mathbb{R} \times Y, X))$ the set of all such functions, where

$$
\begin{aligned}
A A_{0}(X) & :=\left\{f \in B C(\mathbb{R}, X): \lim _{T \rightarrow \infty} \frac{1}{2 T} \int_{-T}^{T}\|f(\sigma)\| \mathrm{d} \sigma=0\right\}, \\
A A_{0}(\mathbb{R} \times Y, X) & :=\left\{f \in B C(\mathbb{R} \times Y, X): \lim _{T \rightarrow \infty} \frac{1}{2 T} \int_{-T}^{T}\|f(\sigma, u)\| \mathrm{d} \sigma\right. \\
& =0 \quad \text { uniformly for } u \text { in any bounded subset of } Y\} .
\end{aligned}
$$

Definition 2.3 ([2]). Let $\mu \in \mathfrak{M}$. A bounded continuous function $f: \mathbb{R} \rightarrow X$ is said to be $\mu$-ergodic if

$$
\lim _{T \rightarrow \infty} \frac{1}{\mu([-T, T])} \int_{[-T, T]}\|f(t)\| \mathrm{d} \mu(t)=0 .
$$

We denote the space of all such functions by $\mathscr{E}(\mathbb{R}, X, \mu)$. 
Definition 2.4. ([2]). Let $\mu \in \mathfrak{M}$. A bounded continuous function $f: \mathbb{R} \rightarrow X$ is said to be $\mu$-pseudo almost automorphic if $f$ is written in the form $f=g+\varphi$, where $g \in A A(\mathbb{R}, X)$ and $\varphi \in \mathscr{E}(\mathbb{R}, X, \mu)$. We denote the space of all such functions by $P A A(\mathbb{R}, X, \mu)$.

Definition 2.5. ([2]). Let $\mu \in \mathfrak{M}$. A bounded continuous function $f: \mathbb{R} \times Y \rightarrow X$ is said to be $\mu$-pseudo almost automorphic if $f$ is written in the form $f=g+\varphi$, where $g \in A A(\mathbb{R} \times Y, X)$ and $\varphi \in \mathscr{E}(\mathbb{R} \times Y, X, \mu)$. We denote the space of all such functions by $P A A(\mathbb{R} \times Y, X, \mu)$, where

$$
\begin{aligned}
& \mathscr{E}(\mathbb{R} \times Y, X, \mu) \\
& \triangleq\left\{f \in B C(\mathbb{R} \times Y, X): \lim _{T \rightarrow \infty} \frac{1}{\mu([-T, T])} \int_{[-T, T]}\|f(t, u)\| \mathrm{d} \mu(t)=0\right. \\
&\text { uniformly for } u \text { in any bounded subset of } Y\} .
\end{aligned}
$$

For $\mu \in \mathfrak{M}$ and $\tau \in \mathbb{R}$, we denote $\mu_{\tau}$ as the positive measure on $\mathfrak{B}$ defined by

$$
\mu_{\tau}(A)=\mu(a+\tau: a \in A), \quad A \in \mathfrak{B} .
$$

For $\mu \in \mathfrak{M}$, we list the following hypothesis ([2]).

(H0) For all $\tau \in \mathbb{R}$, there exist $\gamma>0$ and a bounded interval $I$ such that

$$
\mu_{\tau}(A) \leq \gamma \mu(A),
$$

when $A \in \mathfrak{B}$ satisfies $A \bigcap I=\emptyset$.

Lemma 2.6. [2, Theorem 3.5]. Let $\mu \in \mathfrak{M}$ satisfy (H0). Then $\mathscr{E}(\mathbb{R}, X, \mu)$ is translation invariant; therefore, PAA( $\mathbb{R}, X, \mu)$ is also translation invariant.

Lemma 2.7. [2, Theorem 4.9]. Let $\mu \in \mathfrak{M}$. Assume that $P A A(\mathbb{R}, X, \mu)$ is translation invariant. Then $\left(P A A(\mathbb{R}, X, \mu),\|\cdot\|_{\infty}\right)$ is a Banach space.

Remark 2.8. On examples of measure $\mu$ and functions in $P A A(\mathbb{R}, X, \mu)$, we refer the reader to the reference [2].

Now, let us recall some basic definitions and results on fractional differentiation, integration and resolvent operators. 
Definition 2.9. ([24]). The fractional order integral of a function $f \in L^{1}([0, b], X)$ of order $\alpha \in \mathbb{R}_{+}$is defined by

$$
J_{t}^{\alpha} f(t)=\frac{1}{\Gamma(\alpha)} \int_{0}^{t}(t-s)^{\alpha-1} f(s) \mathrm{d} s,
$$

where $\Gamma$ is the Gamma function.

Definition 2.10. ([24]). The Caputo fractional order derivative of order $\alpha$ of a function $f \in L^{1}([0, b], X)$ given on the interval $[0, b]$ is defined by (if it exists)

$$
D_{t}^{\alpha} f(t)=\frac{1}{\Gamma(n-\alpha)} \int_{0}^{t}(t-s)^{n-\alpha-1} f^{(n)}(s) \mathrm{d} s,
$$

where $\alpha \in(n-1, n], n \in \mathbb{N}$.

Since $f$ takes values in Banach space $X$, the integrals which appear in the above definitions are taken in Bochner's sense.

In the remainder of this paper we always suppose that $0<\alpha<1$, $g_{\alpha}(t)=t^{\alpha-1} / \Gamma(\alpha), t>0$ and $A$ is a closed and densely defined linear operator on $X$.

Definition 2.11. ([25]). A family $\left\{S_{\alpha}(t)\right\}_{t \geq 0} \subseteq \mathscr{L}(X)$ of bounded linear operators in $X$ is called a resolvent (or solution operator) generated by $A$ if the following conditions are satisfied:

(S1) $S_{\alpha}(t)$ is strong continuous on $\mathbb{R}_{+}$and $S_{\alpha}(0)=I$;

(S2) $S_{\alpha}(t) D(A) \subseteq D(A)$ and $A S_{\alpha}(t) x=S_{\alpha}(t) A x$ for all $x \in D(A)$ and $t \geq 0$

(S3) the resolvent equation holds for

$$
S_{\alpha}(t) x=x+\int_{0}^{t} g_{\alpha}(t-s) A S_{\alpha}(s) x \mathrm{~d} s \quad \text { for all } x \in D(A), t \geq 0 .
$$

3. Composition theorems. In this section, we will establish compositions of $\mu$-pseudo almost automorphic functions under suitable conditions by using pseudo almost automorphic functions and measure theory. 
Denote by $L^{p}(\mathbb{R}, \mu)$ the space of real-valued integrable functions on $\mathbb{R}$ with the measure $\mu \in \mathfrak{M}$ and the norm

$$
\|f\|_{L^{p}(\mu)}=\left(\int_{\mathbb{R}}|f(t)|^{p} \mathrm{~d} \mu(t)\right)^{1 / p},
$$

where $1 \leq p<\infty$.

We start with the following characterization of $\mu$-ergodic functions in terms of the measure $\mu$.

Lemma 3.1. [2, Theorem 2.14]. Let $\mu \in \mathfrak{M}$ and $I$ be a bounded interval (eventually $I=\emptyset$ ). Assume that $f \in B C(\mathbb{R}, X)$. Then the following assertions are equivalent:

(i) $f \in \mathscr{E}(\mathbb{R}, X, \mu)$.

(ii)

$$
\lim _{T \rightarrow \infty} \frac{1}{\mu([-T, T] \backslash I)} \int_{[-T, T] \backslash I}\|f(t)\| \mathrm{d} \mu(t)=0 .
$$

(iii) For any $\varepsilon>0$,

$$
\lim _{T \rightarrow \infty} \frac{\mu(\{t \in[-T, T] \backslash I:\|f(t)\|>\varepsilon\})}{\mu([-T, T] \backslash I)}=0 .
$$

Theorem 3.2. Let $f=g+h \in P A A(\mathbb{R} \times X, X, \mu)$ with $g \in A A(\mathbb{R} \times$ $X, X)$ and $h \in \mathscr{E}(\mathbb{R} \times X, X, \mu)$. Assume:

(i) $g(t, x)$ is uniformly continuous on any bounded subset $\Omega \in X$ uniformly in $t \in \mathbb{R}$.

(ii) There exists a nonnegative function $l \in L^{p}(\mathbb{R}, \mu)(1 \leq p<\infty)$ such that

$$
\|f(t, x)-f(t, y)\| \leq l(t)\|x-y\|
$$

for all $x, y \in X$ and $t \in \mathbb{R}$.

Then $f(\cdot, \phi(\cdot)) \in P A A(\mathbb{R}, X, \mu)$ provided that $\phi \in P A A(\mathbb{R}, X, \mu)$.

Proof. Let

$$
\phi(t)=\alpha(t)+\beta(t),
$$

where $\alpha \in A A(\mathbb{R}, X)$ and $\beta \in \mathscr{E}(\mathbb{R}, X, \mu)$. Since

$$
f(t, x)=g(t, x)+h(t, x),
$$


where $g \in A A(\mathbb{R} \times X, X)$ and $h \in \mathscr{E}(\mathbb{R} \times X, X, \mu)$, we have

$$
\begin{aligned}
f(t, \phi(t)) & =g(t, \alpha(t))+f(t, \phi(t))-g(t, \alpha(t)) \\
& =g(t, \alpha(t))+f(t, \phi(t))-f(t, \alpha(t))+h(t, \alpha(t)) .
\end{aligned}
$$

Denote

$$
\begin{aligned}
G(t) & =g(t, \alpha(t)), \\
H(t) & =f(t, \phi(t))-f(t, \alpha(t))+h(t, \alpha(t)) .
\end{aligned}
$$

In view of [19, Lemma 2.2], we have $G \in A A(\mathbb{R}, X)$.

To prove that $f(\cdot, \phi(\cdot)) \in P A A(\mathbb{R}, X, \mu)$, it is enough to show that $H \in \mathscr{E}(\mathbb{R}, X, \mu)$.

Firstly, we show that $f(\cdot, \phi(\cdot))-f(\cdot, \alpha(\cdot)) \in \mathscr{E}(\mathbb{R}, X, \mu)$. It is easy to see that $f(\cdot, \phi(\cdot))-f(\cdot, \alpha(\cdot))$ is bounded and continuous on $\mathbb{R}$. We can assume that $\| f(t, \phi(t)-f(t, \alpha(t)) \| \leq M$, for all $t \in \mathbb{R}$. Since $\beta \in \mathscr{E}(\mathbb{R}, X, \mu)$, Lemma 3.1 yields that, for any $\varepsilon>0$,

$$
\lim _{T \rightarrow \infty} \frac{\mu(\{t \in[-T, T]:\|\beta(t)\|>\varepsilon\})}{\mu([-T, T])}=0 .
$$

Thus,

$$
\begin{aligned}
& \frac{1}{\mu([-T, T])} \int_{[-T, T]}\|f(t, \phi(t))-f(t, \alpha(t))\| \mathrm{d} \mu(t) \\
= & \frac{1}{\mu([-T, T])} \int_{\{t \in[-T, T]:\|\beta(t)\|>\varepsilon\}}\|f(t, \phi(t))-f(t, \alpha(t))\| \mathrm{d} \mu(t) \\
& +\frac{1}{\mu([-T, T])} \int_{[-T, T] \backslash\{t \in[-T, T]:\|\beta(t)\|>\varepsilon\}}\|f(t, \phi(t))-f(t, \alpha(t))\| \mathrm{d} \mu(t) \\
\leq & \frac{M}{\mu([-T, T])} \mu(\{t \in[-T, T]:\|\beta(t)\|>\varepsilon\}) \\
& +\frac{\varepsilon}{\mu([-T, T])} \int_{[-T, T]} l(t) \mathrm{d} \mu(t) .
\end{aligned}
$$

If $p=1$, we have

$$
\frac{\varepsilon}{\mu([-T, T])} \int_{[-T, T]} l(t) \mathrm{d} \mu(t) \leq \frac{\varepsilon\|l\|_{L^{1}(\mu)}}{\mu([-T, T])} .
$$


If $p>1$, we have

$$
\begin{aligned}
& \frac{\varepsilon}{\mu([-T, T])} \int_{[-T, T]} l(t) \mathrm{d} \mu(t) \\
& \leq \frac{\varepsilon}{\mu([-T, T])}\left(\int_{[-T, T]} l^{p}(t) \mathrm{d} \mu(t)\right)^{1 / p}\left(\int_{[-T, T]} \mathrm{d} \mu(t)\right)^{1 / q} \\
& \leq \frac{\varepsilon\|l\|_{L^{p}(\mu)}}{(\mu([-T, T]))^{1-1 / q}},
\end{aligned}
$$

where $q=p(1-p)^{-1}$. Hence, we have

$$
\lim _{T \rightarrow \infty} \frac{1}{\mu([-T, T])} \int_{[-T, T]}\|f(t, \phi(t))-f(t, \alpha(t))\| \mathrm{d} \mu(t)=0,
$$

which implies that $f(\cdot, \phi(\cdot))-f(\cdot, \alpha(\cdot)) \in \mathscr{E}(\mathbb{R}, X, \mu)$.

Secondly, we show that $h(\cdot, \alpha(\cdot)) \in \mathscr{E}(\mathbb{R}, X, \mu)$. Let $\varepsilon>0$. Since $g$ satisfies condition (i), there exists a number $\delta>0$ such that

$$
\|g(t, x)-g(t, y)\| \leq \varepsilon,
$$

for all $t \in \mathbb{R}, x, y \in X$ with $\|x-y\| \leq \delta$.

Put $\delta_{0}=\min \{\varepsilon, \delta\}$. Then

$$
\begin{aligned}
\|h(t, x)-h(t, y)\| & \leq\|f(t, x)-f(t, y)\|+\|g(t, x)-g(t, y)\| \\
& \leq[l(t)+1] \varepsilon
\end{aligned}
$$

for all $t \in \mathbb{R}, x, y \in X$ with $\|x-y\| \leq \delta_{0}$.

Since $K=\overline{\{\alpha(t): t \in \mathbb{R}\}}$ is compact, one can find a finite open ball $O_{k}(k=1,2, \ldots, m)$ with center $x_{k} \in K$ and radius $\delta_{0}$ small enough such that

$$
\{\alpha(t): t \in \mathbb{R}\} \subset \bigcup_{k=1}^{m} O_{k} .
$$

And, we choose $B_{k}$ such that

$$
B_{k}=\left\{s \in \mathbb{R}: \alpha(s) \in O_{k}\right\}, \quad \mathbb{R}=\bigcup_{k=1}^{m} B_{k} .
$$

Let

$$
E_{1}=B_{1}, \quad E_{k}=B_{k} \backslash \bigcup_{j=1}^{k-1} B_{j} \quad(2 \leq k \leq m) .
$$


Then

$$
E_{i} \bigcap E_{j}=\emptyset, \quad i \neq j, 1 \leq i, j \leq m
$$

Define the step function $\bar{x}: \mathbb{R} \rightarrow X$ by $\bar{x}(s)=x_{k}, s \in E_{k}, k=$ $1,2, \ldots, m$. It is easy to see that $\|\alpha(s)-\bar{x}(s)\| \leq \delta_{0}$ for all $s \in \mathbb{R}$. By (3.1), it follows that

$$
\begin{aligned}
& \frac{1}{\mu([-T, T])} \int_{[-T, T]} \| h(t, \alpha(t) \| \mathrm{d} \mu(t) \\
= & \frac{1}{\mu([-T, T])} \int_{\cup_{k=1}^{m}\left(E_{k} \cap[-T, T]\right)} \| h(t, \alpha(t) \| \mathrm{d} \mu(t) \\
\leq & \frac{1}{\mu([-T, T])} \sum_{k=1}^{m} \int_{E_{k} \cap[-T, T]}[\| h(t, \alpha(t)-h(t, \bar{x}(t)) \| \\
\leq & \frac{1}{\mu([-T, T])} \sum_{k=1}^{m} \int_{E_{k} \cap[-T, T]}[l(t)+1] \varepsilon \mathrm{d} \mu(t) \\
+ & \left.\frac{1}{\mu([-T, T])} \sum_{k=1}^{m} \int_{E_{k} \cap[-T, T]}\left\|h\left(t, x_{k}\right)\right\| \mathrm{d} \mu(t)\right) \| \mathrm{d} \mu(t) \\
\leq & +\frac{\varepsilon}{\mu([-T, T])} \int_{[-T, T]} l(t) \mathrm{d} \mu(t) \\
+ & \frac{1}{\mu([-T, T])} \sum_{k=1}^{m} \int_{[-T, T]}\left\|h\left(t, x_{k}\right)\right\| \mathrm{d} \mu(t) .
\end{aligned}
$$

Using the same arguments as above and the fact $h \in \mathscr{E}(\mathbb{R} \times X, X, \mu)$, we obtain that

$$
\lim _{T \rightarrow \infty} \frac{1}{\mu([-T, T])} \int_{[-T, T]} \| h(t, \alpha(t) \| \mathrm{d} \mu(t)=0,
$$

which implies that $h(\cdot, \alpha(\cdot)) \in \mathscr{E}(\mathbb{R}, X, \mu)$. This completes the proof.

If $p=1$, we can make use of the ergodic boundedness of function $l$ instead of $l \in L^{1}(\mathbb{R}, \mu)$. In fact, we have the following result.

Theorem 3.3. Let $f=g+h \in P A A(\mathbb{R} \times X, X, \mu)$ with $g \in A A(\mathbb{R} \times$ $X, X)$ and $h \in \mathscr{E}(\mathbb{R} \times X, X, \mu)$. Assume: 
(i) $g(t, x)$ is uniformly continuous on any bounded subset $\Omega \in X$ uniformly in $t \in \mathbb{R}$.

(ii) There exist a positive number $M$ and a nonnegative function $l: \mathbb{R} \rightarrow \mathbb{R}$ such that

$$
\frac{1}{\mu([-T, T])} \int_{[-T, T]} l(t) \mathrm{d} \mu(t) \leq M
$$

and

$$
\|f(t, x)-f(t, y)\| \leq l(t)\|x-y\|,
$$

for all $T>0, x, y \in X$ and $t \in \mathbb{R}$.

Then $f(\cdot, \phi(\cdot)) \in P A A(\mathbb{R}, X, \mu)$, provided that $\phi \in P A A(\mathbb{R}, X, \mu)$.

Proof. The proof is similar to that of Theorem 3.2. So, we omit it.

Remark 3.4. The above composition theorems generalize those in $[2,3,18,19]$ by Blot et al., and Liang et al., respectively, where the composition theorems of pseudo almost automorphic functions and weighted pseudo almost automorphic functions are established.

4. $\mu$-pseudo almost automorphic solutions. This section is devoted to the existence of $\mu$-pseudo almost automorphic solutions of the following fractional differential equation in a Banach space $X$ :

$$
D_{t}^{\alpha} x(t)=A x(t)+J_{t}^{1-\alpha} f(t, x(t)), \quad t \in \mathbb{R},
$$

where $0<\alpha<1, A: D(A) \subseteq X \rightarrow X$ generates a resolvent $S_{\alpha}(t), D_{t}^{\alpha}$ is the Caputo fractional derivative and

$$
J_{t}^{1-\alpha} h(t)=\int_{0}^{t}(t-s)^{-\alpha} h(s) / \Gamma(1-\alpha) \mathrm{d} s .
$$

By a mild solution of (4.1), we understand a function $x \in C(\mathbb{R}, X)$ such that (see [25])

$$
x(t)=\int_{-\infty}^{t} S_{\alpha}(t-s) f(s, x(s)) \mathrm{d} s, \quad t \in \mathbb{R} .
$$

For our purposes, we need the following assumptions. 
(H1) The resolvent $S_{\alpha}(t)$ is exponentially stable, that is, there exist $M>0, \omega>0$, such that $\left\|S_{\alpha}(t)\right\| \leq M \exp ^{-\omega t}$ for all $t \geq 0$.

(H2) $f=g+h \in P A A(\mathbb{R} \times X, X, \mu)$ satisfies conditions (i) and (ii) in Theorem 3.2.

Theorem 4.1. Assume conditions (H1) and (H2) hold and $l \in L^{p}(\mathbb{R})$. Then equation (4.1) has a unique $\mu$-pseudo almost automorphic mild solution.

Proof. Consider the nonlinear operator $\mathscr{F}$ given by

$$
(\mathscr{F} x)(t)=\int_{-\infty}^{t} S_{\alpha}(t-s) f(s, x(s)) \mathrm{d} s .
$$

In view of condition (H2) and Theorem 3.2, we obtain that $f(\cdot, x(\cdot)) \in$ $P A A(\mathbb{R}, X, \mu)$ if $x \in P A A(\mathbb{R}, X, \mu)$. Thus, according to [2, Theorem $3.9]$, we can see that $\mathscr{F}$ maps $P A A(\mathbb{R}, X, \mu)$ into itself.

Firstly, let $l \in L^{1}(\mathbb{R}, \mu)$, and we have

$$
\begin{aligned}
\|(\mathscr{F} x)(t)-(\mathscr{F} y)(t)\| & \leq \int_{-\infty}^{t}\left\|S_{\alpha}(t-s)[f(s, x(s))-f(s, y(s))]\right\| \mathrm{d} s \\
& \leq M\|x-y\| \int_{-\infty}^{t} l(s) \mathrm{d} s
\end{aligned}
$$

and

$$
\begin{aligned}
\left\|\left(\mathscr{F}^{2} x\right)(t)-\left(\mathscr{F}^{2} y\right)(t)\right\| & \leq M \int_{-\infty}^{t} l(s)\|(\mathscr{F} x)(s)-(\mathscr{F} y)(s)\| \mathrm{d} s \\
& \leq M\|x-y\| \int_{-\infty}^{t} l(s) \int_{-\infty}^{s} l(\sigma) \mathrm{d} \sigma \mathrm{d} s \\
& \leq \frac{M^{2}}{2}\|x-y\|\left(\int_{-\infty}^{t} l(s) \mathrm{d} s\right)^{2} .
\end{aligned}
$$

Thus, by induction on $n \geq 1$, we have

$$
\left\|\left(\mathscr{F}^{n} x\right)(t)-\left(\mathscr{F}^{n} y\right)(t)\right\| \leq \frac{M^{n}}{n !}\|x-y\|\left(\int_{-\infty}^{t} l(s) \mathrm{d} s\right)^{n} .
$$


Therefore,

$$
\left\|\mathscr{F}^{n} x-\mathscr{F}^{n} y\right\| \leq \frac{\left(M\|l\|_{L^{1}(\mathbb{R})}\right)^{n}}{n !}\|x-y\|,
$$

which implies that $\mathscr{F}^{n}$ is a contraction for sufficiently large $n$. Hence, there exists a unique fixed point $x \in P A A(\mathbb{R}, X, \mu)$ of operator $\mathscr{F}$ by the generalized contraction mapping principle, which is just the unique $\mu$-pseudo almost automorphic mild solution of (4.1).

Secondly, let $l \in L^{p}(\mathbb{R})(1<p<\infty)$. Put $\lambda(t)=\int_{-\infty}^{t} l^{p}(s) \mathrm{d} s$. Then, we define an equivalent norm over $P A A(\mathbb{R}, X, \mu)$, as follows

$$
\|f\|_{c}=\sup _{t \in \mathbb{R}}\left\|e^{-c \lambda(t)} f(t)\right\|,
$$

where $c$ is a fixed positive number. Then, we have

$$
\begin{aligned}
\|(\mathscr{F} x)(t)-(\mathscr{F} y)(t)\| & \leq \int_{-\infty}^{t}\left\|S_{\alpha}(t-s)[f(s, x(s))-f(s, y(s))]\right\| \mathrm{d} s \\
& \leq \int_{-\infty}^{t} l(s)\left\|S_{\alpha}(t-s)\right\|\|x(s)-y(s)\| \mathrm{d} s \\
& \leq M \int_{-\infty}^{t} e^{-\omega(t-s)} l(s) e^{c \lambda(s)}\|x-y\|_{c} \mathrm{~d} s \\
& \leq M\left(\int_{-\infty}^{t} e^{c p \lambda(s)} l^{p}(s) \mathrm{d} s\right)^{1 / p} \\
& \quad\left(\int_{-\infty}^{t} e^{-\omega q(t-s)} \mathrm{d} s\right)^{1 / q}\|x-y\|_{c} \\
& \leq M(\omega q)^{-1 / q}\left(\int_{-\infty}^{t} e^{c p \lambda(s)} \lambda^{\prime}(s) \mathrm{d} s\right)^{1 / p}\|x-y\|_{c} \\
& \leq M(\omega q)^{-1 / q}(c p)^{-1 / p} e^{c \lambda(t)}\|x-y\|_{c} .
\end{aligned}
$$

Therefore,

$$
\|\mathscr{F} x-\mathscr{F} y\|_{c} \leq M(\omega q)^{-1 / q}(c p)^{-1 / p}\|x-y\|_{c},
$$

which implies that $\mathscr{F}$ is a contraction for sufficiently large $c$. Hence, there exists a unique fixed point $x \in P A A(\mathbb{R}, X, \mu)$ of operator $\mathscr{F}$ by the generalized contraction mapping principle, which is just the unique 
$\mu$-pseudo almost automorphic mild solution of (4.1). This completes the proof.

Remark 4.2. The above results generalize those in $[\mathbf{1}, \mathbf{8}, \mathbf{9}, \mathbf{2 0}$, where the existence of weighted pseudo almost periodic, almost automorphic, compact almost automorphic and pseudo almost automorphic mild solutions to equation (4.1) are derived.

Acknowledgments. The authors are grateful to the referee for valuable comments and suggestions.

\section{REFERENCES}

1. R.P. Agarwal, B. de Andrade and C. Cuevas, Weighted pseudo-almost periodic solutions of a class of semilinear fractional differential equations, Nonlin. Anal. RWA 11 (2010), 3532-3554.

2. J. Blot, P. Cieutat and K. Ezzinbi, Measure theory and pseudo almost automorphic functions: New developments and applications, Nonlin. Anal. TMA 75 (2012), 2426-2447.

3. J. Blot, G.M. Mophou, G.M. N'Guérékata and D. Pennequin, Weighted pseudo almost automorphic functions and applications to abstract differential equations, Nonlin. Anal. TMA 71 (2009), 903-909.

4. S. Bochner, A new approach to almost-periodicity, Proc. Nat. Acad. Sci. 48 (1962), 2039-2043.

5. Y.K. Chang, G.M. N'Guérékata and R. Zhang, Stepanov-like weighted pseudo almost automorphic functions via measure theory, Bull. Malays. Math. Sci. Soc., DOI $10.1007 / \mathrm{s} 40840-015-0206-1$.

6. Y.K. Chang, R. Zhang and G.M. N'Guérékata, Weighted pseudo almost automorphic solutions to nonautonomous semilinear evolution equations with delay and $S^{p}$-weighted pseudo almost automorphic coefficients, Top. Meth. Nonlin. Anal. 43 (2014), 69-88.

7. P. Cieutat, S. Fatajou and G.M. N'Guérékata, Composition of pseudo almost periodic and pseudo almost automorphic functions and applications to evolution equations, Appl. Anal. 89 (2010), 11-27.

8. C. Cuevas and C. Lizama, Almost automorphic solutions to a class of semilinear fractional differential equations, Appl. Math. Lett. 21 (2008), 13151319.

9. C. Cuevas, M. Rabelo and H. Sot, Pseudo-almost automorphic solutions to a class of semilinear fractional differential equations, Comm. Appl. Nonlin. Anal. 17 (2010), 31-47.

10. T. Diagana, Almost automorphic type and almost periodic type functions in abstract spaces, Springer, New York, 2013. 
11. T. Diagana, Existence of pseudo-almost automorphic solutions to some abstract differential equations with $S^{p}$-pseudo-almost automorphic coefficients, Nonlin. Anal. TMA 70 (2009), 3781-3790.

12. T. Diagana and G.M. N'Guérékata, Stepanov-like almost automorphic functions and applications to some semilinear equations, Appl. Anal. 86 (2007), 723733 .

13. T. Diagana, G.M. Mophou and G.M. N'Guérékata, Existence of weighted pseudo-almost periodic solutions to some classes of differential equations with $S^{p}$ weighted pseudo-almost periodic coefficients, Nonlin. Anal. TMA 72 (2010), 430438.

14. H.S. Ding, J. Liang and T.J. Xiao, Some properties of Stepanov-like almost automorphic functions and applications to abstract evolution equations, Appl. Anal. 88 (2009), 1079-1091.

15. Almost automorphic solutions to nonautonomous semilinear evolution equations in Banach spaces, Nonlin. Anal. TMA 73 (2010), 1426-1438.

16. Z.B. Fan, J. Liang and T.J. Xiao, On Stepanov-like (pseudo) almost automorphic functions, Nonlin. Anal. TMA 74 (2011), 2853-2861.

17. Composition of Stepanov-like pseudo almost automorphic functions and applications to nonautonomous evolution equations, Nonlin. Anal. RWA 13 (2012), 131-140.

18. J. Liang, G.M. N'Guérékata, T.J. Xiao and J. Zhang, Some properties of pseudo-almost automorphic functions and applications to abstract differential equations, Nonlin. Anal. TMA 70 (2009), 2731-2735.

19. J. Liang, J. Zhang and T.J. Xiao, Composition of pseudo almost automorphic and asymptotically almost automorphic functions, J. Math. Anal. Appl. 340 (2008), 1493-1499.

20. C. Lizama and F. Poblete, Regularity of mild solutions for a class of fractional order differential equations, Appl. Math. Comp. 224 (2013), 803-816.

21. G.M. N'Guérékata, Almost automorphic and almost periodic functions in abstract spaces, Kluwer Academic/Plenum Publishers, New York, 2001.

22. , Topics in almost automorphy, Springer, New York, 2005.

23. G.M. N'Guérékata and A. Pankov, Stepanov-like almost automorphic functions and monotone evolution equations, Nonlin. Anal. TMA 68 (2008), 2658-2667.

24. I. Podlubny, Fractional differential equations, Academic Press, San Diego, 1999.

25. J. Prüss, Evolutionary integral equations and applications, Birkhäuser, Basel, 1993.

26. Z.N. Xia and M. Fan, Weighted Stepanov-like pseudo almost automorphy and applications, Nonlinear Anal. TMA 75 (2012), 2378-2397.

27. T.J. Xiao, J. Liang and J. Zhang, Pseudo almost automorphic solutions to semilinear differential equaitons in Banach spaces, Semigroup Forum 76 (2008), $518-524$. 
28. T.J. Xiao, X.X. Zhu and J. Liang, Pseudo-almost automorphic mild solutions to nonautonomous differential equations and applications, Nonlin. Anal. TMA 70 (2009), 4079-4085.

School of Mathematics, Yangzhou University, Yangzhou, Jiangsu 225002, P.R. China

Email address: zbfan@yzu.edu.cn, jsmathfan@gmail.com

School of Mathematics, Yangzhou University, Yangzhou, Jiangsu 225002, P.R. CHINA

Email address: qxdongyz@outlook.com

School of Mathematics, Yangzhou University, Yangzhou, Jiangsu 225002, P.R. CHINA

Email address: gli@yzu.edu.cn 